\title{
On uniqueness of analytic solution for first order partial differential equations with degenerate principal symbols
}

\author{
By
}

Akira NAKAOKA

(Communicated by Prof. Mizohata May 28, 1974)

\section{Introduction}

In the previous paper [5], the author studied the uniquess of analytic solution of the Cauchy problem to the following equation with initial data on the hyperplane $t=0$;

$$
\begin{aligned}
& {\left[\begin{array}{cc}
E_{N-1} & 0 \\
0 & 0
\end{array}\right] \frac{\partial \vec{u}}{\partial t}} \\
& \quad=\sum_{j=1}^{n}\left[\begin{array}{ll}
B_{j}(t, x) & K_{j}(t, x) \\
L_{j}(t, x) & \phi^{j}(t, x)
\end{array}\right]_{\partial x_{j}}^{\partial \vec{u}}+\left[\begin{array}{cc}
C(t, x) & P(t, x) \\
0 & \alpha(t, x)
\end{array}\right] \vec{u},
\end{aligned}
$$

where $E_{N-1}$ denotes the $(N-1) \times(N-1)$ unit matrix, $B_{j}(t, x)$ 's and $C(t, x)$ are $(N-1) \times(N-1)$, and $K_{j}(t, x)$ 's, ${ }^{t} L_{j}(t, x)$ 's and $P(t, x)$ are $(N-1) \times 1$ matrices with entries of analytic fnnctions of $(t, x)$ in a neighborhood of the origin, and finally $\phi^{j}(t, x)$ 's are analytic functions in a neighborhood of the origin. We called (1.1) of thpe $(N-1, N-1)$ if $\alpha(0,0) \neq 0$ (for the general definition of type $(p, q)$, see Definition. 1.1 of [5]).

In [5] the author treated the case of $\alpha(t, x) \equiv-1$, and obtained the following results;

Theorem 1. A necessary condition for the solution of the 
Cauchy problem for (1.1) to be unique is

$$
\phi^{j}(0,0)=0 \quad(j=1,2, \cdots, n) .
$$

Theorem 2. If the solution, which is analytic in a neighborhood of $x=0$, of the following equation

$$
\sum_{j=1}^{n} \phi^{j}(0, x) \partial f / \partial x_{j}=f
$$

is only trivial one, then the solution of lhe Cauchy problem for (1.1) is unique. Moreover, if for any n-tuples of non-negative integers $\left(\alpha_{1}, \cdots, \alpha_{n}\right)$ it holds

$$
\alpha_{1} \lambda_{1}+\cdots+\alpha_{n} \lambda_{n} \neq 1
$$

then the equation (1.2) has no analytic solution except the trivial one, where $\lambda_{j}$ 's are the eigenvalues of $\left(\partial \phi^{j}(0,0) / \partial x_{k}\right)(j, k=1, \cdots, n)$.

However, we can give a simple example of the equation which has only trivial solution, nevertheless the equation (1.3) is violated by some $n$-tuples of non-negative integers $\left(\alpha_{1}, \cdots, \alpha_{n}\right)$ (see the 4 -th example of section 6).

In this paper, we shall consider the case when $\alpha(0, x)$ vanishes at $x=0$, but exclude the case when $\alpha(0, x) \equiv 0$. Moreover, we are limited to the case of $n=2$, that is, our equation concerned is as follows ;

$$
a(x, y) \partial u / \partial x+b(x, y) \partial u / \partial y=c(x, y) u .
$$

In addition to the analyticity of $a(x, y), b(x, y)$ and $c(x, y)$ in a neighborhood of the origin, we assume the followings throughout this paper;

(H. 1) $\partial^{p+q} c(0,0) / \partial x^{p} \partial y^{q}=0$ for all non-negative integers $p$ and $q$ such that $p+q \leqq m-1$ with some integer $m$ larger than 1 , and $\partial^{m} c(0,0) / \partial x^{m}=1$ (we may assume this without loss of generality). (H. 2) $\quad \partial^{p+q} a(0,0) / \partial x^{p} \partial y^{q}=\partial^{p+q} b(0,0) / \partial x^{p} \partial y^{q}=0$ for all non-negative integers $p$ and $q$ such that $p+q \leqq m$.

The purpose of this paper is to find sufficient conditions under which the analytic solution in a neighborhood of the origin of the equation (1.4) to be only zero. Such a problem, of course, is deeply 
related to the question whether or not the equation (1.4) admits an analytic integral in some neighborhood of the origin. For this, in the early days $\mathrm{H}$. Poincare already investigated the case when $\alpha(0, x)$ $\equiv-1$ essentially and the number of variables is general $n$, in his thesis [8], and obtained a sufficient condition for the integrability.

Recently, T. Oshima [6] obtained a result related to these problems. In [6] he studied the equation of $n$ variables not assuming (H.2), and weakening hypothesis of [8] partly but under some additional algebraic conditions for the coefficicients, he determined Coker $P$ and Ker $P$, where $P$ denotes the concerning operator. However, the result of [6] does not cover fully those of [5] or of this paper, and conversely the result of [5] or of this paper does not fully the result of [6] as for the uniqueness of the solution. For example, our result is not applicable to the 11-th example of [6].

Our principle in order that the analytic solution of (1.4) is only zero is to show that the all derivatives of the solution vanish at the origin. And we use the term "solution" only for the analytic solution throughout this paper, since we are only concerned with the analytic solution.

In section 2 , we study the structure of the coefficient matrices of the equations which determine the values at the origin of the partial derivatives of the solution of (1.4) of easc order.

In the third section, we shall consider the case when the coefficient matrices considered in section 2 are of triangular type, and the case which we can make them to those of triangular type by a suitable linear transformation (equivalently by an analytic transformation) of independent variables with kept the hypothesis (H.1). We say such an equation "triangularizable".

In the fourth section, we shall show that the equation (1.4) has no solution except the trivial one, unless (1.4) is essentially triangularizable.

In section 5 , we shall slightly make mention of the case in which the hypothesis (H.2) is removed. There, we shall show that the methods used in the previous sections are also applicable to the equation when the vanishing orders of $a(x, y), b(x, y)$ and $c(x, y)$ at the origin are same, and the final section is devoted to giving 
some examples.

From the next section to the forth section, we use the notations $A=A(x, y), B=B(x, y)$, and $C=C(x, y)$ for the homogeneous parts of degree $m+1$ of $a(x, y)$ and $b(x, y)$ and for the homogeneous part of $m$ of $c(x, y)$ respectively. By virtue of (H.2), all the arguments in these sections will be carried out in terms of $A, B$ and $C$.

At the end of this section, we claim that if the vanishing orders of $a(x, y)$ and $b(x, y)$ both are larger than that of $c(x, y)$ plus two, then the equation (1.4) has no non-trivial solution.

\section{Structure of coefficient matrices}

In this section, we study the structure of the coefficient masrices of the systems of the equations which determine the values at the origin of the partial derivatives of the solution of (1.4) of each order.

Let us rewrite the equation (1.4) as follows;

$$
C(x, y) u=A(x, y) \partial u / \partial x+B(x, y) \partial u / \partial y+R(u ; x, y),
$$

where $R(u ; x, y)$ is given by

$$
(a-A) \partial u / \partial x+(b-B) \partial u / \partial y+(C-c) u .
$$

Differentiating $m$ times with respect to $x$ the both sides of (2.1), we have

$$
\begin{aligned}
\sum_{l=0}^{m}{ }_{m} C_{l} \cdot C^{(m-l)} u^{(l)}= & \sum_{l=0}^{m}{ }_{m} C_{l} \cdot A^{(m-l)} u^{(l+1)}+\sum_{l=0}^{m}{ }_{m} C_{l} B^{(m-l)} u_{(1)}^{(l)} \\
& +R^{(m)}(u ; x, y),
\end{aligned}
$$

where $f_{(q)}^{(p)}$ stands for $\partial^{p+q} f / \partial x^{p} \partial y^{q}$ for a function $f(x, y)$ and ${ }_{m} C_{l}$ is defined for any integer $m$ and $l$ by

$$
{ }_{m} C_{l}=\left\{\begin{array}{cl}
m ! /(m-l) ! l ! & \text { if } m \geqq l \geqq 0 \\
0 & \text { if } m<0 \text { or } l<0 \text { or } m<l .
\end{array}\right.
$$

From (H.2) and (2.2), we have at first $u(0,0)=0$, and it is easily seen that $R(u ; x, y)$ gives no influence to determine the values of the partial derivatives at the origin of order $n+1$, if all the derivatives of order less than $n$ vanish at the origin.

The main aim of this section is to prove the following two 
theorems.

Theorem 2. 1. Let $\omega(x, y)$;

$$
\omega(x, y)=\sum_{l=0}^{\mu} \omega_{l} x^{\mu-l} y^{l} \quad\left(\omega_{0}=1\right),
$$

be the greatest common divisor of $A(x, y), B(x, y)$ and $C(x, y)$ in $C[x, y]$, and $\vec{U}_{N}$ be the column vector whose $(k+1)$-th component is given by $\partial^{N} u(0,0) / \partial x^{N-k} \partial y^{k}(k=0,1, \cdots, N)$ for each non-negative integer $N$, then for the equation to determine $\vec{U}_{N}$ from $\vec{U}_{0}, \vec{U}_{1}, \cdots$, $\vec{U}_{N-1}$;

$$
S_{N}\left(\omega_{1}, \cdots, \omega_{\mu}\right) \vec{U}_{N}=F_{N}\left(\vec{U}_{0}, \vec{U}_{1}, \cdots, \vec{U}_{N-1}\right),
$$

where $S_{N}\left(\omega_{1}, \cdots, \omega_{\mu}\right)$ is a $(N+1) \times(N+1)$ matrix which depends on $A, B$, and $C$, and which we regard as a function of $\left(\omega_{1}, \cdots, \omega_{\mu}\right)$, in particular, and $\vec{F}_{N}$ is a vector with $N+1$ components depending on $\vec{U}_{0}, \vec{U}_{1}, \cdots, \vec{U}_{N-1}$ linearly, we see that the determinant of $S_{N}\left(\omega_{1}, \cdots\right.$, $\left.\omega_{\mu}\right)$ is independent of $\left(\omega_{1}, \cdots, \omega_{\mu}\right)$ for each $N$.

Theorem 2.2. For each $N$, the kernel of $S_{N}\left(\omega_{1}, \cdots, \omega_{\mu}\right)$ does not depend on $\left(\omega_{1}, \cdots, \omega_{\mu}\right)$.

In order to prove above theorems, we prepare a few lemmas. Let us put

$$
\left\{\begin{array}{l}
A(x, y)=\omega(x, y) \alpha(x, y) \\
B(x, y)=\omega(x, y) \beta(x, y) \\
C(x, y)=\omega(x, y) \gamma(x, y),
\end{array}\right.
$$

and

$$
\left\{\begin{array}{l}
\alpha(x, y)=\sum_{\nu=0}^{m-\mu} \alpha_{\nu} x^{m+1-\mu-\nu} y^{\nu} \\
\beta(x, y)=\sum_{\nu=0}^{m-\mu} \beta_{\nu} x^{m+1-\mu-\nu} y^{\nu} \\
\gamma(x, y)=\sum_{\nu=0}^{m-\mu} \gamma_{\nu} x^{m-\mu-\nu} y^{\nu} \quad\left(\gamma_{0}=1\right) .
\end{array}\right.
$$

We begin with 
Lemma 2. 1. It holds

$$
(N-p) \sum_{q}{ }_{N} C_{q} \cdot{ }_{K} C_{q-p}=(K+p+1) \sum_{q}{ }_{N} C_{q} \cdot{ }_{K} C_{q-p-1} .
$$

Proof. Notice that

$$
(N-p){ }_{N} C_{q}=(q-p){ }_{N} C_{q}+(q+1){ }_{N} C_{q+1},
$$

when $N \geqq q+1$ and $q \geqq p$. In fact, we have

$$
\begin{aligned}
(q-p){ }_{N} C_{q}+(q+1){ }_{N} C_{q+1} & =(q-p) N ! /(N-q) ! q !+N ! /(N-q-1) ! q ! \\
& =(N-p){ }_{N} C_{q},
\end{aligned}
$$

and hence we obtain

$$
\begin{aligned}
(N-p) \sum_{q}{ }_{N} C_{q} \cdot{ }_{K} C_{q-p} & =\sum_{q}\left\{(q-p){ }_{N} C_{q}+(q+1){ }_{N} C_{q+1}\right\}_{K} C_{q-p} \\
& =\sum_{q}\left\{q \cdot{ }_{K} C_{q-p-1}+(q-p){ }_{K} C_{q-p}\right\}_{N} C_{q} \\
& =(K+p+1) \sum_{q}{ }_{N} C_{q} \cdot{ }_{K} C_{q-p-1} .
\end{aligned}
$$

Lemma 2. 2. The left hand side of the $(k+1)$-th equation of (2.4) is given by

$$
\begin{aligned}
\sum_{\sigma} \sum_{l}{ }_{m} C_{l} \cdot{ }_{N-k} C_{l-\sigma}(m-\sigma) !\left\{{ } _ { k } C _ { \sigma } \sigma ! \left((N+\sigma-k) \sum_{\tau} \omega_{\sigma-\tau} \alpha_{\tau}\right.\right. \\
\left.\left.-\sum_{\tau} \omega_{\sigma-\tau} \gamma_{\tau}\right)+{ }_{k} C_{\sigma+1}(\sigma+1) ! \sum_{\tau} \omega_{\sigma+1-\tau} \beta_{\tau}\right\} U_{N}{ }^{k-\sigma},
\end{aligned}
$$

where $U_{N}{ }^{j}$ denotes the $(j+1)-t h$ component of $\vec{U}_{N}$, and $\alpha_{\tau}=\beta_{\tau}=0$, if $\tau<0$ or $\tau>m+1, \gamma_{\tau}=0$ if $\tau<0$ or $\tau>m$ and $\omega_{\tau}=0$ if $\tau<0$ or $\tau>\mu$.

Proof. After inserting (2.5) into (2.1) and differentiating $m$ times with respect to $x$ both sides, we have

$$
\begin{aligned}
\sum_{l}{ }_{m} C_{l}(\omega \gamma)^{(m-l)} u^{(l)}= & \sum_{l}{ }_{m} C_{l}\left\{(\omega \alpha)^{(m-l)} u^{(l)}\right. \\
& \left.+(\omega \beta)^{(m-l)} u_{(1)}^{(l)}\right\}+R^{(m)}(u ; x, y),
\end{aligned}
$$

and $(N-k)$ times wit respect to $x$ further, we get

$$
\sum_{\sigma} \sum_{l}{ }_{m} C_{l} \cdot{ }_{N-K} C_{l-\sigma}(\omega \gamma)^{(m-\sigma)} u^{(N-k+\sigma)}
$$




$$
\begin{aligned}
& =\sum_{\sigma} \sum_{l}{ }_{m} C_{l} \cdot{ }_{N-k} C_{l-\sigma}\left\{(\omega \alpha)^{(m-\sigma)} u^{(N+1-k+\sigma)}\right. \\
& \left.\quad+(\omega \beta)^{(m-\sigma)} u_{(1)}^{(N-k+\sigma)}\right\}+R^{(m+N-k)}(u ; x, y),
\end{aligned}
$$

and moreover, $k$ times with respect to $y$, we obtain

$$
\begin{aligned}
& \sum_{\tau} \sum_{\sigma} \sum_{l}{ }_{m} C_{l} \cdot{ }_{N-k} C_{l-\sigma} \cdot{ }_{k} C_{\tau}(\omega \gamma)_{(\tau)}^{(m-\sigma)} u_{(k-\tau)}^{(N+k+\sigma)} \\
& =\sum_{\tau} \sum_{\sigma} \sum_{l}{ }_{m} C_{l} \cdot{ }_{N-k} C_{l-\sigma} \cdot{ }_{k} C_{\tau}\left\{(\omega \alpha)_{(\tau)}^{(m-\sigma)} u_{(k-\tau)}^{(N-k+1+\sigma)}\right. \\
& \left.\quad+(\omega \beta)_{(\tau)}^{(m-\sigma)} u_{(k+1-\tau)}^{(N-k+\sigma)}\right\}+R_{(k)}^{(m+N-k)}(u ; x, y) .
\end{aligned}
$$

Now let $x=y=0$ in (2.11), then we obtain

$$
\begin{aligned}
\sum_{\sigma} \sum_{l}{ }_{m} C_{l} \cdot{ }_{N-k} C_{l-\sigma}(\omega \gamma) \underset{(\sigma)}{(m-\sigma)} U_{N}{ }^{k-\sigma} \\
=\sum_{\sigma} \sum_{l}{ }_{m} C_{l} \cdot{ }_{N-k} C_{l-\sigma} \cdot{ }_{k} C_{\sigma+1}\left\{(\omega \alpha)_{(\sigma+1)}^{(M-\sigma)} U_{N}{ }^{k-\sigma-1}\right. \\
\left.\quad+(\omega \beta) \underset{(\sigma+1)}{(m-\sigma)} U_{N}{ }^{k-\sigma}\right\}+R_{(k)}^{(m+N-k)}(u ; 0,0),
\end{aligned}
$$

where $R_{(k)}^{(m+N-k)}(u ; 0,0)$ is represented in terms of $\vec{U}_{0}, \vec{U}_{1}, \cdots, \vec{U}_{N-1}$, and hence

$$
\begin{aligned}
& \sum_{\sigma} \sum_{l}{ }_{m} C_{l}\left({ }_{k} C_{\sigma}\left\{{ }_{N-k} C_{l+1-\sigma}(\omega \alpha) \underset{(\sigma)}{(m+1-\sigma)}-{ }_{N-k} C_{l-\sigma}(\omega \gamma) \underset{(\sigma)}{(m-\sigma)}\right\}\right. \\
& +{ }_{k} C_{\sigma+1} \cdot{ }_{N-k} C_{l-\sigma}(\omega \beta) \underset{(\sigma+1)}{(m-\sigma)} U_{N}{ }^{k-\sigma}=-R_{(k)}^{(m+N-k)}(u ; 0,0),
\end{aligned}
$$

Now let us notice

$$
\begin{aligned}
& (\omega \alpha))_{(\sigma)}^{(m+1-\alpha)}=(m+1-\sigma) ! \sigma ! \sum_{\tau} \omega_{\sigma-\tau} \alpha_{\tau} \\
& (\omega \beta)_{(\sigma+1)}^{(m-\sigma)}=(m-\sigma) !(\sigma+1) ! \sum_{\tau} \omega_{\sigma-\tau} \beta_{\tau} \\
& (\omega \gamma)_{(\sigma)}^{(m+\sigma)}=(m-\sigma) ! \sigma ! \sum_{\tau} \omega_{\sigma-\tau} \gamma_{\tau},
\end{aligned}
$$

and

$$
(m+1-\sigma) \sum_{l}{ }_{m} C_{l} \cdot{ }_{N-k} C_{l+1-\sigma}=(N-k+\sigma) \sum_{l}{ }_{m} C_{l} \cdot{ }_{N-k} C_{l-\sigma},
$$

which follows from Lemma. 2.1, then we obtain (2.8) if we insert (2.14) and (2.15) into (2.13), and the $(k+1)$-th component of $F_{N}\left(U_{0}, U_{1}, \cdots, U_{N-1}\right)$ is given by $-R_{(k)}^{(m+N-k)}(u ; 0,0)$. Q.E.D.

The following lemma is important. 
Lemma 2. 3. Let $\vec{s}_{k+1}$ be the $(k+1)$-th row vector of $S_{N}\left(\omega_{1}, \cdots\right.$, $\left.\omega_{\mu}\right)$, then we have

$$
\partial s_{k+1} /\left.\partial \omega_{\lambda} \sim s_{k+1-\lambda}\right|_{\omega_{1}=\cdots=\omega_{\nu}=0}
$$

where $\nu=\min (\mu, k-\lambda)$ and $\sim i$ s the notation to denote that the vectors of both sides are proportional eace other, and here we assume $\vec{s}_{j}=0$ if $j \leqq 0$.

Proof. Let us consider the case when $\nu=k-\lambda$. The proof of the case when $\nu=\mu$ is carried out similarly. We notice that the coefficient of $\omega_{\lambda}$ in the $j$-th component of $\vec{s}_{k+1}$ is given by

$$
\begin{aligned}
& k ! /(j-1) ! \sum_{l}{ }_{m} C_{l} \cdot{ }_{N-k} C_{l-k+j-1}(m-k+j-1) ! \\
& \quad \times\left\{(N-j+1) \alpha_{k-j-\lambda+1}-\gamma_{k-j+\lambda+1}+(j-1) \beta_{k-j-\lambda+2}\right\},
\end{aligned}
$$

and the $j$-th component of $\left.\vec{s}_{k+1-\lambda}\right|_{\omega_{1}=\ldots=\omega_{k-\lambda}=0}$ is given

$$
\begin{gathered}
(k-\lambda) ! /(j-1) ! \sum_{l}{ }_{m} C_{l} \cdot{ }_{N+\lambda-k} C_{l-k+\lambda-1+j}(m-k+\lambda-1+j) ! \\
\quad \times\left\{(N-j+1) \alpha_{k-j-\lambda+1}-\gamma_{k-j-\lambda+1}+(j-1) \beta_{k-j-\lambda+1}\right\} .
\end{gathered}
$$

Now, using Lemma. 2.1 respectedly, we have

$$
\begin{aligned}
(m- & k+\lambda-1+j) ! \sum_{l}{ }_{m} C_{l} \cdot{ }_{N+\lambda-k} C_{l-k+\lambda-1+j} \\
= & (N+2-j)(N+3-j) \cdots(N-1)(m-k+\lambda) ! \\
& \quad \times \sum_{l} C_{l} C{ }_{N+\lambda-k} C_{l-k+\lambda},
\end{aligned}
$$

and

$$
\begin{aligned}
& (m-k+j-1) ! \sum_{l}{ }_{m} C_{l} \cdot{ }_{N-k} C_{l-k+j-1} \\
& \quad=(N+2-j)(N+3-j) \cdots(N-1)(m-k) ! \sum_{l} C_{l} C_{N_{N-k}} C_{l-k},
\end{aligned}
$$

hence we obtain

$$
\begin{aligned}
& (k-\lambda) !(m-k+\lambda) ! \sum_{l}{ }_{m} C_{l} \cdot{ }_{N+\lambda-k} C_{l-k+\lambda} \partial \vec{s}_{k+1} / \partial \omega_{\lambda} \\
& =\left.k !(m-k) ! \sum_{l} C_{l} C_{N-k} C_{l-k} \vec{s}_{k+1-\lambda}\right|_{\omega_{1}=\cdots=\omega_{k-\lambda}=0} .
\end{aligned}
$$

This implies (2.16). Q.E.D. 
Now we prove the following proposition, then Theorem. 2.1 and Theorem. 2.2 can be obtained as its cororally.

Proposition 2. 1. For each $N, S_{N}\left(\omega_{1}, \cdots, \omega_{\mu}\right)$ can be decomposed as follows;

$$
S_{N}\left(\omega_{1}, \cdots, \omega_{\mu}\right)=T_{N}\left(\omega_{1}, \cdots, \omega_{\mu}\right) S_{N}(0, \cdots, 0),
$$

where $T_{N}\left(\omega_{1}, \cdots, \omega_{\mu}\right)$, a matrix of order $N+1$, is as follows;

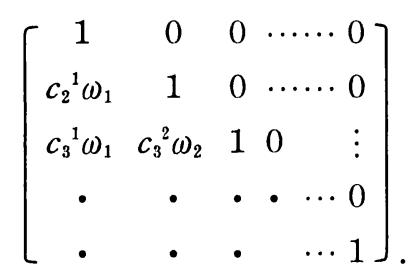

Proof. From (2.8) we see that each $\vec{s}_{\text {, }}$ is given by a linear function of $\left(\omega_{1}, \cdots \omega_{\mu}\right)$, and by virtue of (2.16) of Lemma. 2.3, we have

$$
\begin{aligned}
& \vec{s}_{2}=c_{2}{ }^{1} \omega_{1} \vec{s}_{1}+\vec{s}_{2}(0), \\
& \vec{s}_{j}=\sum_{\nu=1}^{\mu} c_{j}{ }^{\nu} \omega_{\nu} \vec{s}_{j-\nu}(0, \cdots, 0)+\vec{s}_{j}(0, \cdots, 0),
\end{aligned}
$$

with some suitable constants $c_{j}{ }^{\nu}$. This proves our assertion. Q.E.D.

\section{Triangularizable case}

In this section, we shall treat the case in which each $S_{N}=S_{N}(\omega$, $\left.\cdots, \omega_{\mu}\right)$ is of triangular type or which we can triangularize it by a suitable transformation of independent variables.

We start with

Lemma 3. 1. The matrices $S_{N}(N=1,2, \cdots)$ are of triangular type if and only if so is $S_{1}$, that is to say, $\partial^{m+1} B(0,0) / \partial x^{m+1}=0$.

Proof. It suffices to note (2.8). Q.E.D.

At first we have

Theorem 3. 1. Let $\partial^{m+1} B(0,0) / \partial x^{m+1}=0$. If for all non-nega- 
tive integers $j$ and $k$, it holds

$$
j \alpha_{0}+k \beta_{1} \neq 1,
$$

then the solntion of (1.4) does not exist except the trivial one.

Proof. We prove this theorem by showing thal all the derivatives of the solution of (1.4) vanish at the origin. For this, we use the mathematical induction with respect to the order of derivatives. Here, we follow the notations of the previous section. Since $\vec{U}_{0}=\overrightarrow{0}$, we we have $S_{1} \vec{U}_{1}=0$, and the equation (3.1) with $j+k=1$ implies that $\operatorname{det} S_{1} \neq 0$. So we obtain $\vec{U}_{1}=\overrightarrow{0}$. Now suppose here $\vec{U}_{j}=\overrightarrow{0} \quad(j=1$, $2 \cdots, N-1$ ), then we have $S_{N} \vec{U}_{N}=\overrightarrow{0}$. Therefore, the equation (3.1) with $j+k=N$ implies $\operatorname{det} S_{N} \neq 0$, and hence $\vec{U}_{N}=\overrightarrow{0}$. This completes the proof. Q.E.D.

The next to be considered is the case in which $\partial^{m+1} B(0,0) / \partial x^{m+1}$ does not necessarily vanish. In this case, we aim to reduce the equation to the case when $\partial^{m+1} B(0,0) / \partial x^{m+1}=0$ by suitable transformation of independent variables. In doing so, it suffices to consider only a linear transformation, since we are concerned with the values at the origin, that is to say,

$$
\left[\begin{array}{l}
x \\
y
\end{array}\right]=\left[\begin{array}{ll}
p & q \\
r & s
\end{array}\right]\left[\begin{array}{l}
\xi \\
\eta
\end{array}\right] \quad(p q \neq r s) .
$$

After the transformation (3.2), the equation (1.4) turns into

$$
a^{*}(\xi, \eta) \partial u / \partial \xi+b^{*}(\xi, \eta) \partial u / \partial \eta=c^{*}(\xi, \eta) u,
$$

where $a^{*}(\xi, \eta), b^{*}(\xi, \eta)$ and $c^{*}(\xi, \eta)$ are given by

$$
\begin{aligned}
& a^{*}(\xi, \eta)=\Delta^{-1}\{s a(p \xi+q \eta, r \xi+s \eta)-q b(p \xi+q \eta, r \xi+s \eta)\} \\
& b^{*}(\xi, \eta)=\Delta^{-1}\{r a(p \xi+q \eta, r \xi+s \eta)+p b(p \xi+q \eta, r \xi+s \eta)\} \\
& c^{*}(\xi, \eta)=c(p \xi+q \eta, r \xi+s \eta)
\end{aligned}
$$

where $\Delta=p s-q r$.

Definition 3.1. The equation (1.4) is said to be triangularizable if and only if a suitable choice of $p, q, r$ and $s$ with $p s \neq q r$ implies $\partial^{m} c^{*}(0,0) / \partial \xi^{m}=1$ and $\partial^{m+1} b^{*}(0,0) / \partial \xi^{m+1}=0$. 
We have

Theorem 3.2. The equation (1.4) is triangularizable if and only if the following system of algebraic equations;

$$
\left\{\begin{array}{l}
x B(x, y)-y A(x, y)=0 \\
C(x, y)=1
\end{array}\right.
$$

has a root.

Proof. It suffices to note

$$
\begin{aligned}
& \partial^{m+1} b^{*}(0,0) / \partial \xi^{m}=\Delta^{-1}\{p B(p, r)-r A(p, r)\} \\
& \partial^{m} c^{*}(0,0) / \partial \xi^{m}=C(p, r) . \quad \text { Q.E.D. }
\end{aligned}
$$

Remark. If (3.5) has a root, we can choose $q$ and s such as $\Delta \neq 0$, since $(0,0)$ is not the rootof $(3.5)$.

By Theorem. 3.1, we obtain

Theorem 3. 3. Let $A(x, y)=\omega(x, y) \alpha(x, y), B(x, y)=\omega(x, y)$ $\beta(x, y)$ and $C(x, y)=\omega(x, y) \gamma(x, y)$, where $\omega(x, y)$ is the greatest common divisor of $A(x, y), B(x, y)$ and $C(x, y)$. Suppose that the system of the algebraic equations;

$$
\left\{\begin{array}{l}
x \beta(x, y)-y \alpha(x, y)=0 \\
C(x, y)=1,
\end{array}\right.
$$

has a root. If for some root $(p, r)$ of (3.6), these exist two complex numbers $q$ and s satisfying $p s \neq q r$ such that for all nonnegative integers $j$ and $k$

$$
j \lambda+k \mu \neq p s-q r,
$$

where $\lambda$ and $\mu$ are given by

$$
\lambda=s \alpha(p, r)-q \beta(p, r),
$$

and

$$
\begin{aligned}
\mu= & -r\{q \partial \alpha(p, r) / \partial x+s \partial \alpha(p, r) / \partial y\} \\
& +p\{q \partial \alpha(p, r) / \partial x+s \partial \beta(p, r) / \partial y\},
\end{aligned}
$$


then the solution of (1.4) is only zero.

Proof. For such $p, q, r$ and $s$, consider the linear transformation given by (3.2), and then we can see that the equation (3.3) has no solution except the trivial one by Theorem. 3.1, and so does not the equation (1.4).

Theorem. 3. 1 and Theorem. 3. 3 describe the sufficient condition under which the diterminant of $S_{N}$ never vanishes for each $N$, when $S_{N}$ is of triangular type or can be reduced by a suitable exchange of independent variable. If the determinant of $S_{N}$ vanishes for some $N$, we must impose some conditions on the higher order terms of the expansion of $a(x, y), b(x, y)$ and $c(x, y)$ to obtain the uniqueness of the solution.

\section{Non-triangularizable case}

In this section we will cosider the equation which is not triangularizable. So we assume here that the equation (3.5) has no root, that is, all $(x, y)$ satifying

$$
x B(x, y)-y A(x, y)=0
$$

satisfies also

$$
C(x, y)=0 .
$$

Let $A(x, y), B(x, y)$ and $C(x, y)$ be as of (2.5). At first, we consider the case when the following system of algebraic equations;

$$
\left\{\begin{array}{l}
x \beta(x, y)-y \alpha(x, y)=0 \\
\gamma(x, y)=1
\end{array}\right.
$$

has a root $(p, r)$. Then, we may assume that the following matrix $S_{1}$ is of triangular type with $\partial^{m+1-\mu} \beta(0,0) / \partial x^{m+1-\mu}=0$ by taking the linear transformation of independent variables given by (3.2);

$$
S_{1}=\left[\begin{array}{ll}
\partial^{m+1-\mu} \alpha(0,0) / \partial x^{m+1-\mu} & \partial^{m+1-\mu} \beta(0,0) / \partial x^{m+1-\mu} \\
\partial^{m+1-\mu} \alpha(0,0) / \partial x^{m-\mu} \partial y & \partial^{m+1-\mu} \beta(0,0) / \partial x^{m-\mu} \partial y
\end{array}\right] .
$$

Thus we obtain 
Theorem 4.1. Suppase the equation (4.1) has a root, and hence $\partial^{m+1-\mu} \beta(0,0) / \partial x^{m+1-\mu}=0$. If for all non-negative integers $j$ and $k$, the equation (3.1) holds, then the solution of (1.4) is only zero.

Proof. Let $U_{k}$ be the homogeneous part of degree $k$ of the solution. The proof can be carried out by the induction with respect to $k$. Obviously $U_{0}=0$ and let $U_{1}=\cdots=U_{k-1}=0$, then $U_{k}$ satisfies

$$
\omega \gamma(x, y) U_{k}=\omega \alpha(x, y) \partial U_{k} / \partial x+\omega \beta(x, y) \partial U_{k} / \partial y .
$$

Since $\omega(x, y) \not \equiv 0$, dividing both sides by $\omega(x, y)$, we can apply the same argument as of the proof of Theorem. 3.1. Q.E.D.

The case whice we must cosider is when the equation (4.1) has no root. Then, taking an exchange of independent variables, we may assume $\gamma(x, y)$ has $x$ as its factor. Now let $x, x+c_{1} y, \cdots, x+c_{n} y$ be the irreducible factors of $\gamma(x, y)$, and let $\gamma(x, y)$ be as follows;

$$
\gamma(x, y)=x^{\mu_{0}}\left(x+c_{1} y\right)^{\mu_{1}} \cdots\left(x+c_{n} y\right)^{\mu_{n}} .
$$

Since the zeros of $x \beta(x, y)-y \alpha(x, y)$ are all those of $\gamma(x, y)$, we may put

$$
x \beta(x, y)-y \alpha(x, y)=\delta x^{\lambda_{0}}\left(x+c_{1} y\right)^{\lambda_{1}} \cdots\left(x+c_{n} y\right)^{\lambda_{n}},
$$

where $\lambda_{j}$ are all non-negative integers, and $\delta$ a constant. That the equation is not triangularizable assures $\delta \neq 0$.

Before stating our main theorem of this section, we prepare the following easy lemma.

Lemma 4. 1. In (4.4) we have $\lambda_{j} \geqq 2$ for some $j$. Hence we may assume $\lambda_{0} \geqq 2$ after a suitable exchange of independent variables, if necessary.

Proof. Since $x \beta(x, y)-y \alpha(x, y)$ is homogeneous of degree $m+2-\mu$ and it does not vanish identically, we have

$$
\lambda_{0}+\lambda_{1}+\cdots+\lambda_{n}=m+2-\mu .
$$

So if $\lambda_{j} \leqq 1$ for all $j$, we get $m+1-\mu \leqq n$. On the other hand, we have $n+1 \leqq m-\mu$, for the degree of $\gamma(x, y)$ is $m-\mu$. This leads us to a contradiction. Q.E.D. 
By the above lemma, we assume $\lambda_{0} \geqq 2$ hereafter.

The main aim of this section is to establish the following theorem.

Theorem 4.2. Suppose that the equation (4.1) has no root. Then the equation (1.4) has no solution except the trivial one.

Proof. We use the mathematical induction also. Let $U_{k}$ be the homogeneous part of degree $k$ of the solution, and let $U_{1}=\cdots=U_{k-1}$ $=0$. Of course, we know already $U_{0}=0$. Now, then we obtain

$$
\gamma(x, y) U_{k}=\alpha(x, y) \partial U_{k} / \partial x+\beta(x, y) \partial U_{k} / \partial y .
$$

Here, let us put $U_{k}$ as follows;

$$
U_{k}=u_{0} x^{k}+u_{1} x^{k-1} y+\cdots+u_{k-1} x y^{k-1}+u_{k} y^{k},
$$

then (4.5) becomes

$$
\begin{aligned}
& x^{\mu}{ }_{0}\left(x+c_{1} y\right)^{\mu_{1}} \cdots\left(x+c_{n} y\right)^{\mu_{n}}\left(u_{0} x^{k}+\cdots+u_{k-1} x y^{k-1}+u_{k} y^{k}\right) \\
& =\left(\alpha_{0} x^{m+1-\mu}+\alpha_{1} x^{m-\mu} y+\cdots+\alpha_{m-\mu} x y^{m-\mu}+\alpha_{m+1-\mu} y^{m+1-\mu}\right) \\
& \times\left(k u_{0} x^{k-1}+(k-1) u_{1} x^{k-2} y+\cdots+2 u_{k-2} x y^{k-2}+u_{k-1} y^{k-1}\right) \\
& +\left(\beta_{0} x^{m+1-\mu}+\beta_{1} x^{m-\mu} y+\cdots+\beta_{m-\mu} x y^{m-\mu}+\beta_{m+1-\mu} y^{m+1-\mu}\right) \\
& \times\left(u_{1} x^{k-1}+2 u_{2} x^{k-2} y+\cdots+(k-1) u_{k-1} x y^{k-2}+k u_{k} y^{k-1}\right) .
\end{aligned}
$$

On the other hand, observing

$$
\begin{aligned}
x \beta(x, y)-y \alpha(x, y) & \\
= & \beta_{0} x^{m+2-\mu}+\left(\beta_{1}-\alpha_{0}\right) x^{m+1-\mu} y+\cdots+\left(\beta_{m+1-\mu}-\alpha_{m-\mu}\right) x y^{m+1-\mu} \\
& \quad-\alpha_{m+1-\mu} y^{m+2-\mu}
\end{aligned}
$$

and noticing that $x^{2}$ is a factor of $x \beta(x, y)-y \alpha(x, y)$, we obtain $\alpha_{m-\mu}=\beta_{m+1-\mu}$ and $\alpha_{m+1-\mu}=0$. Moreover, we have $\beta_{m+1-\mu} \neq 0$. In fact, if $\beta_{m+1-\mu}=0$, we see that $x$ gives a common factor of $\alpha(x, y), \beta(x, y)$ and $\gamma(x, y)$, and this contradicts to that $\omega(x, y)$ is the greatest common divisor of $A(x, y), B(x, y)$ and $C(x, y)$. Thus (4.7) turns out as follows;

$$
\begin{aligned}
& x^{\mu_{0}}\left(x+c_{1} y\right)^{\mu_{1}} \cdots\left(x+c_{n} y\right)^{\mu_{n}}\left(u_{0} x^{k}+\cdots+u_{k-1} x y^{k-1}+u_{k} y^{k}\right) \\
& =\left(\alpha_{0} x^{m+1-\mu}+\cdots+\alpha_{m-1-\mu} x^{2} y^{m-1-\mu}+\beta_{m+1-\mu} x y^{m-\mu}\right)
\end{aligned}
$$




$$
\begin{aligned}
& \times\left(k \mu_{0} x^{k-1}+(k-1) u_{1} x^{k-2} y+\cdots+2 u_{k-2} x y^{k-2}+u_{k-1} y^{k-1}\right) \\
& +\left(\beta_{0} x^{m+1-\mu}+\cdots+\beta_{m-\mu} x y^{m-\mu}+\beta_{m+1-\mu} y^{m+1-\mu}\right) \\
& \times\left(u_{1} x^{k-1}+2 u_{2} x^{k-2} y+\cdots+(k-1) u_{k-1} x y^{k-2}+k u_{k} y^{k-1}\right) .
\end{aligned}
$$

Since $\mu_{0} \geqq 1$ and $\beta_{m+1-\mu} \neq 0$, we have $u_{k}=0$, and successively $u_{k-1}=0$, $u_{k-2}=0$ and so on. This implies $U_{k}=0$. Q.E.D.

\section{The case when the vanishing orders of $a(x, y), b(x, y)$ and} $c(x, y)$ are same

In this section, we shall treat the case when the vanishing orders af $a(x, y), b(x, y)$ and $c(x, y)$ at the origin are same. That is, in addition to (H.1), we assume here the following in place of (H.2); (H. 2)' $\partial^{p+q} a(0,0) / \partial x^{p} \partial y^{q}=\partial^{p+q} b(0,0) / \partial x^{p} \partial y^{q}=0$ for all non-negative integars $p$ and $q$ such that $p+q \leqq m-1 \quad(m \geqq 1)$.

To avoid any trouble about notations, let us use the different notations from the previous sections. Let us denote by $f(x, y), g(x, y)$ and $h(x, y)$ the homogeneous parts of degree $m$ of $a(x, y), b(x, y)$ and $c(x, y)$ respectively. And by $\Omega(x, y)$, we denote the greatest common divisor of $f(x, y)$ and $g(x, y)$, and we set

$$
\left\{\begin{array}{l}
f(x, y)=\Omega(x, y) F(x, y) \\
g(x, y)=\Omega(x, y) G(x, y) .
\end{array}\right.
$$

We obtain the following theorem.

Theorem 5.1. Suppose that

(1) if a triple of complex numbers $\left(\kappa_{1}, \kappa_{2}, \kappa_{3}\right)$ satisfies

$$
\kappa_{1} f(x, y)+\kappa_{2} g(x, y)=\kappa_{3} h(x, y),
$$

it follows $\kappa_{3}=0$.

(2) $x G(x, y)-y F(x, y) \equiv 0 \bmod (\lambda x+\mu y)^{2}$, for some $(\lambda, \mu) \neq(0,0)$. Then the solution of (1.4) is only zero.

Proof. Let us denote by $U_{k}$ the homogeneous part of degree $k$ of the solution. First we have

$$
f(x, y) \partial U_{1} / \partial x+g(x, y) \partial U_{1} / \partial y=h(x, y) U_{0},
$$

and in virtue of (1) we obtain $U_{0}=0$. Thus, we get 


$$
F(x, y) \partial U_{1} / \partial x+G(x, y) \partial U_{1} / \partial y=0 .
$$

Now let $F(x, y)$ and $G(x, y)$ be as follows;

$$
\begin{aligned}
& F(x, y)=F_{0} x^{n}+\cdots+F_{n-1} x y^{n-1}+F_{n} y^{n}, \\
& G(x, y)=G_{0} x^{n}+\cdots+G_{n-1} x y^{n-1}+G_{n} y^{n}
\end{aligned}
$$

We claim here that none of $F(x, y)$ and $G(x, y)$ vanishes identically. Indeed, let, for example, $G(x, y) \equiv 0$, then we have $\Omega(x, y)=f(x, y)$ and consequently $F(x, y) \equiv 1$, and this contradicts to (2). Moreover, we notice that we may assume $(\lambda, \mu)=(1,0)$ by considering the following transformation of independent variables;

$$
\begin{aligned}
& \xi=\lambda x+\mu y \\
& \eta=\tilde{\lambda} x+\tilde{\mu} y \quad(\lambda \tilde{\mu} \neq \tilde{\lambda} \mu) .
\end{aligned}
$$

Thus we have $F_{n}=0$ and $G_{n}=F_{n-1}$. Moreover, we see $G_{n} \neq 0$, if ${ }_{x} G(x, y)-y F(x, y)$ does not vanish identically. In fact, if $G_{n}=0$, $F(x, y)$ and $G(x, y)$ have $x$ as their common foctor, and this contradicts to the assumption on them. If $x G(x, y)-y F(x, y)$ vanishes identically, we have easily $F(x, y)=x$ and $G(x, y)=y$. We first take the case when $x G(x, y)-y F(x, y) \not \equiv 0$.

Let $U_{1}=u_{1} x+u_{2} y$, then we obtain from (5.3)

$$
u_{1}\left(F_{0} x^{n}+\cdots+F_{n-2} x^{2} y^{n-2}+G_{n} x y^{n-1}\right)+u_{2}\left(G_{0} x^{n}+\cdots+G_{n} y^{n}\right)=0,
$$

and this implies $u_{1}=u_{2}=0$ since $G_{n} \neq 0$. Now let $U_{1}=U_{2}=\cdots=U_{k-1}=0$, then as is easily seen, we obtain the following equation of $U_{k}$;

$$
F(x, y) \partial U_{k} / \partial x+G(x, y) \partial U_{k} / \partial y=0 .
$$

Here, let us put

$$
U_{k}=u_{0} x^{k}+u_{1} x^{k-1} y+\cdots+u_{k-1} x y^{k-1}+u_{k} y^{k},
$$

and inserting this into (5.6), we have

$$
\begin{aligned}
& \left(F_{0} x^{n}+\cdots+F_{n-2} x^{2} y^{n-2}+G_{n} x y^{n-1}\right)\left(k u_{0} x^{k-1}+(k-1) u_{1} x^{k-2} y\right. \\
& \left.+\cdots+u_{k-1} y^{k-1}\right)+\left(G_{0} x^{n}+\cdots+G_{n} y^{n}\right)\left(u_{1} x^{k-1}+2 u_{2} x^{k-2} y\right. \\
& \left.+\cdots+(k-1) u_{k-1} x y^{k-2}+k u_{k} y^{k-1}\right)=0 .
\end{aligned}
$$

Hence the same arguments as of the proof of Theorem. 4.2 leads us 
to $U_{k}=0$. The remainder part of the proof is the case when $F(x, y)$ $=x$ and $G(x, y)=y$. But if we notice (5.7), we can easily show $U_{k}=0$ for this case. Q.E.D.

The next object of our studies is the case when (2) is removed. Then, we assume that the degrees of $F(x, y)$ and $G(x, y)$ are larger than 1. Under such assupmption on $F(x, y)$ and $G(x, y)$, we see that $x G(x, y)-y F(x, y)$ has at least two irreducible factors, since the polynomial of the form $(\lambda x+\mu y)^{2}$ can not be the factor of $x G(x, y)-y F(x, y)$. Therefore we may assume that $x G(x, y)$ $-y F(x, y)$ has $x$ and $y$ as its factors, considering the following linear transformation of independent variables;

$$
\begin{aligned}
& \xi=\sigma_{1} x+\sigma_{2} y \\
& \eta=\tau_{1} x+\tau_{2} y,
\end{aligned}
$$

where $\sigma_{1} x+\sigma_{2} y$ and $\tau_{1} x+\tau_{2} y$ are distinct irreducible factors of $x G(x, y)$ $-y F(x, y)$. Let us set

$$
x G(x, y)-y F(x, y)=J x y\left(x+d_{1} y\right) \cdots\left(x+d_{s} y\right),
$$

where $J$ is a non-zero constant and $d_{1}, \cdots, d_{s}$ are non-zero and dicinct constants. Of course $\left(x+d_{1} y\right) \cdots\left(x+d_{s} y\right)$ does not appear if the degree of $F(x, y)$ (and consequently of $G(x, y)$ ) is equal to 1 . From (5.8) can see immediately that $F(x, y)$ has $x$ as its factor and $G(x, y)$ has $y$ as its factor. Thus we obtain $F_{n}=0$ and $G_{0}=0$, and therefore $F_{0} \neq 0$ and $G_{n} \neq 0$ since $F(x, y)$ and $G(x, y)$ have no common factor.

We obtain the following theorem.

Theorem 5. 2. Suppose that (1) is valid. If the following relation hold for any natural numbers $N$ and $\nu$;

$$
N F_{0}+\nu J \neq 0
$$

then the solution of (1.4) is only zero, Moreover, if the degree of $F(x, y)$ and $G(x, y)$ are larger than 2, (5.9) can be replaced by

$$
N G_{n}+\nu d_{1} \cdots d_{s} J \neq 0
$$


Proof. We use the same notations as those of the proof of Theorem. 5.1. We must show that all $U_{k}$ 's vanish identically. Clearly $U_{0}=0$ holds, and hence we have (5.3) also. Thus we get $U_{1}=0$ since $F(x, y)$ and $G(x, y)$ are linearly independent because $F_{0} \neq 0$ and $G_{n} \neq 0$.

Now let $U_{1}=U_{2}=\cdots=U_{k-1}=0$, then we have (5.6) also, and therefore we obtain

$$
\begin{aligned}
& \left(F_{0} x^{n}+\cdots+F_{n-1} x y^{n-1}\right)\left(k u_{0} x^{k-1}+(k-1) u_{1} x^{k-2} y+\cdots+u_{k-1} y^{k-1}\right) \\
& \quad+\left(G_{1} x^{n-1} y+\cdots+G_{n} y^{n}\right)\left(u_{1} x^{k-1}+\cdots+(k-1) u_{k-1} x y^{k-2}\right. \\
& \left.\quad+k u_{k} y^{k-1}\right)=0 .
\end{aligned}
$$

Since $F_{0} \neq 0$ and $G_{n} \neq 0$, we see immediately $u_{0}=0$ and $u_{k}=0$, and have

$$
\begin{aligned}
& \left(F_{0} x^{n-1}+\cdots+F_{n-1} y^{n-1}\right)\left((k-1) u_{1} x^{k-2}+\cdots+u_{k-1} y^{k-2}\right) \\
& \quad+\left(G_{1} x^{n-1}+\cdots+G_{n} y^{n-1}\right)\left(u_{1} x^{k-2}+\cdots+(k-1) u_{k-1} y^{k-1}\right)=0 .
\end{aligned}
$$

Thus we obtain

$$
\left((k-1) F_{0}+G_{1}\right) u_{1}=0 .
$$

On the other hand, we have

$$
G_{1}-F_{0}=J
$$

from (5.8). So te have $u_{1}=0$ by (5.9). Now let $u_{1}=u_{2}=\cdots=u_{p}$ $=0$, then we obtain from $(5.12)$

$$
\left((k-p-1) F_{0}+(k+1) G_{1}\right) u_{p+1}=0,
$$

and hence by (5.9) we have $u_{p+1+1}=0$.

The proof of latter part is essentially same. Q.E.D.

If we remove one of (1) and (2) or the condition that the degree of $F(x, y)$ or of $G(x, y)$ is larger than 1 , then we can find the equations which admit null solutions. For this we shall give some examples in the next section.

\section{Examples}

in this section we shall give various examples admitting null 
solutions because the assumptions of our theorems are not satisfied fully.

The first example is of that (1) is dropped.

Example 1. Consider the following exuation;

$$
x^{2} \partial u / \partial x+y^{2} \partial u / \partial y=\left(x^{2}+y^{2}\right) u,
$$

then, as is easily seen, $u(x, y)=e^{x+y}$ gives a null solution.

The second example is of that (2) is dropped and the degree of $F(x, y)$ is equal to zero.

Example 2. Consider the following equation;

$$
T(x, y) \partial u / \partial x+y^{2} \partial u / \partial y=x^{2} u,
$$

where $T(x, y)=x^{3}-2 y^{3}+x^{2} y^{2}$, then $u(x, y)=x+y^{2}$ gives a null solution.

The third example is of that (5.9) is violated.

Example 3. Consider the following equation

$$
x^{2} \partial u / \partial x+x y \partial u / \partial y+y^{3} \partial u / \partial y=y^{2} u,
$$

then $u(x, y)=x y$ gives a null solution.

The final example shows us there exists an equation which admits no solution except zero, even if the determinant of $S_{N}$ vanishes for some $N$.

Example 4. Consider the following equation;

$$
x^{2} \partial u / \partial x+(x+y) \partial u / \partial y=u,
$$

then we can show the solution of (6.4) is only zero. In fact, let us consider the formal solution $u(x, y)$ expanded into Hartogs series;

$$
u(x, y) \sim \sum u_{n}(y) x^{n},
$$

then each $u_{n}(y)$ must satisfy 


$$
y u_{0}^{\prime}(y)=u_{0}(y),
$$

$$
y u_{1}^{\prime}(y)+u_{0}^{\prime}(y)=u_{1}(y)
$$

and

$$
y u_{n+1}^{\prime}(y)+u_{n}^{\prime}(y)+n u_{n}(y)=u_{n+1}(y) \quad(n=1,2, \cdots),
$$

where' stands for the differentiation with respect to $y$.

Hence we have from (6.6) and (6.7),

$$
u_{0}(y)=c_{0} y
$$

and

$$
u_{1}(y)=c_{1} y+c_{0}
$$

where $c_{0}$ and $c_{1}$ are constants. Inserting these into (6.8), we get $c_{1}=0$ and $u_{2}(y)=c_{0}$ and successively $u_{n}(y)=c_{n}$ (constant), since each $u_{n}(y)$ must be analytic at $y=0$. Moreover by $(6.8)$ we have

$$
c_{n}=(n-1) ! c_{0}(n=1,2, \cdots) .
$$

Thus the formal solution becomes

$$
u(x, y) \sim c_{0}\left(x+y+\mathcal{L}(n-1) ! x^{n}\right),
$$

therfore, if $c_{0} \neq 0$, the formal solution never converges in any neighborhood of the origin, and this implies that the solution of (6.6) must be identically zero.

\section{DEPARTMENT OF MATHEMATICS Kyoto TeChNicAl University}

\section{References}

[1] E. Goursat, Cours d'analyse, Paris, 1927.

[2] S. Mizohata, Solutions nulles et solutions non analytiques, J. Math. Kyoto Univ. vol. 1, No. 2, (1962) 271-302.

[3] T. Miwa, On the existence of hyperfunction solution of linear differential equations of first order with degenerate real principal symbols. Proc. Japan Acad. vol. 49, No. 2, (1973) 88-93.

[4] A. Nakaoka, Uniqueness of the solution of some characteristic Cauchy problems for first order systems, Proc. Japan Acad. vol. 49, No. 7, (1973) 520-522.

[5] A. Nakaoka, On uniqueness in some characteristic Cauchy problem for first order systems, J. Math. Kyoto Univ. vol. 14, No. 2, (1974) 319-340. 
[6] T. Oshima, On the theorem of Cauchy-Kowalevsky for first order linear differential equations with degenerate principal symbols, Proc. Japan Acad. vol. 49, No. 2, (1973) 83-87.

[7] E. Picard, Traite d'analyse, Paris, 1922-1928.

[8] H. Poincaré, Sur les propriétés des functions définies par les équations aus différences partielles, Thèses, Oeuures de Henri Poincaré. Paris, 1951. 